\title{
ANALYSIS OF FUNCTIONAL REFERENCE ARCHITECTURE THROUGH AN INDUSTRY LENS
}

\author{
Mactaggart, Ivan Mervyn; \\ Eckert, Claudia; \\ Lockett, Helen
}

The Open University

\begin{abstract}
There is an inherent tension between functional descriptions of products and structural descriptions. Traditionally system architecture combine the two, by mapping system elements to functions. In this process fundamental decisions about the embodiment of a product are often taken without proper scrutiny. Axiomatic design advocates a zigzag between functional decomposition and system break down. While this approach makes sense for ab initio design these are rare and most products are developed incrementally., This paper takes up the idea of a functional reference architecture.

Conventional functional modelling advocates a hierarchical decomposition into sub function. By contrast FRAs decompose function into function chain, i.e., dependent sequences of sub function required to carry out an overall function. This allows the identification of common sub-functions in different chains, and thereby generates a lattice structure of functions rather than a tree. This enables a detailed but solution neutral description of the product.

This concept has attracted interest in industry but does not have many tools and methods to develop FRA. The paper discusses some of the areas that require research
\end{abstract}

Keywords: Design methodology, Systems Engineering (SE), Functional modelling, Complexity, Architecture

\section{Contact:}

Mactaggart, Ivan Mervyn

The Open University

Engineering \& Innovation

United Kingdom

ivan.mactaggart@open.ac.uk 


\section{INTRODUCTION}

There is an inherent tension between functional descriptions of products and structural descriptions. Traditionally system architecture combines the two, by mapping system elements to functions. In this process fundamental decisions about the embodiment of a product are often taken without proper scrutiny. Axiomatic design (e.g., Suh, 2001) is advocating a zigzag between functional decomposition and system break down, developing the details in tandem. While this approach makes sense for ab initio design these are rare and most product are developed incrementally, i.e., you have detailed functional and structural models of part of the system.

The paper takes up the idea of a functional reference architecture (Telleschi, 2018, Caroni, 2019). Conventional functional modelling advocates a hierarchical decomposition into subfunctions. By contrast functional reference architectures decompose function into function chains, i.e., dependent sequences of subfunction required to carry out an overall function. This allows the identification of common sub-functions in different chains, and thereby generates a lattice structure of functions rather than a tree. This enables a detailed but solution neutral description of the product. For many products, the functional architecture remains the same through different embodiments, so that it becomes a reference architecture that is reused and also facilitates reuse in the product. This concept has attracted interest in industry including the companies that the first author has worked in, but there are not many tools and methods to develop functional reference architectures.

The paper discusses functional reference architectures in the context of function modelling and systems engineering and presents a worked example of a reference functional architecture for a hairdryer to illustrate the potential benefits and limitations of the approach.

\section{LITERATURE LANDSCAPE}

The purpose of engineering design is to create a concept for a system or product that can be manufactured. This section identifies the extensive use of models in engineering to create these concepts, and how models are ever increasingly influencing the practice of systems engineering, before turning attention to the specific challenge of modelling function and how function in turn, influences the embodiment through the system architecture. Finally, we explore the notion of Reference Architectures and their emerging use to aid design decision-making in industry practice.

\subsection{Models in engineering}

Engineers use models as representations either expressed in a physical sense (e.g., clay, or wood), or in a document, or digitally (Gollaglee et al., 2020). Models are the way engineers evaluate alternative solutions (Weilkiens, 2006), interact with products, share, and store information. Engineers across all engineering domains use different models, even though the relationship between them is not entirely clear. Within Systems Engineering models are used as the principle means of information transfer and are seen by some as part of a wider drive toward the digitisation of engineering processes to reduce complexity in the design, development, and in-service support of systems (INCOSE, 2015). This approach has become known as Model-based Systems Engineering (MBSE).

\subsection{Models in Systems Engineering}

The systems engineer working in industry is no longer concerned with whether they should model, but how they model (Holt \& Perry, 2013) and, how much they model. The statistician George Box (1919 2013) is often credited with the aphorism "all models are wrong, but some are useful" (Box, 1976, 1979). Whilst Box was referring to statistical models, his observations are equally applicable to the practice of MBSE. Specifically, Box's concern was that because of inaccuracies inherent in any model, elaboration of the model will not lead to increasing precision and knowing when to stop modelling is a critical skill. Identifying a suitable model granularity and assuring that different aspects of a product are modelled to a similar degree of granularity is one of the challenges the system engineering modelling community has not fully resolved. Maier et al. (2016). Hand (2014) observed the aim of modelling is to understand, predict, summarize, or evidence something about the real world, or to help decision-making.

Increased expectation and complexity are driving the accumulative importance of modelling complex systems to improve overall functionality and operability; aesthetics and reduce time to market to 
improve overall affordability and value (e.g., Friedenthal et al., 2012, Holt \& Perry, 2013). Krob (2017) makes clear many design mistakes are made by systems engineers who forget that their system model is not the reality of the "real" system but is an abstraction. Compelling factors driving both new businesses to adopt MBSE, and others to transform from traditional approaches to MBSE have been widely published (e.g., Friedenthal et al., (2012), Holt \& Perry, (2013), Weilkiens, (2006), INCOSE, (2014, 2015); Madni \& Purohit, (2019)). All broadly identify a common set of factors that need to be realized for successful MBSE implementation:

- $\quad$ Early defect detection

- Model and data re-use

- Improved communication between stakeholders

- Improvements in supply chain efficiency

- Product line definition

- Conformance to standards

Whilst existing MBSE approaches support some of these factors, there are still some challenges to achieving effective data reuse and product line definition.

\subsection{Challenge of modelling function}

Modelling is more than the setting out of the concept of the embodiment of the products. Engineers need to consider the purpose of the product, what it is for, and the behaviour required of the product, and its function. Function is significant in engineering design, describing an embodiment neutral description of the system (Eckert, 2013). In the engineering design community extensive research has focussed on function. Crilly (2013) argues the notion of function is core to design but can be interpreted in multiple ways. Definitions of function were extensively analysed by Vermaas (2009, 2010, and 2013) suggesting multiple meanings of function might be mutually supportive rendering any single definition counterproductive. There is a general consensus that functions are solution neutral descriptions of a product, that can be broken down into considerable detail. Many researchers, such as Pahl and Beitz (2007) advocate that solution neutrality should be maintained as long as possible when breaking functions down into subfunctions. However, this can be difficult in practise as subfunction depend on the embodiment decisions taken. Therefore, modelling approaches, such as FBS (Gero and Kannengieser, 2004) see functional modelling as an interactive approach. Axiomatic design (Suh, 2001) explicitly advocates a co-evolution between functional description and embodiment decisions.

The need to model function is widely accepted and practised; however significant issues arise from the varied meanings of function Eckert, (2013). Eisenbart et al. (2013) argue a better understanding of functional modelling approaches is required to guide designers more effectively during the conceptual phases of systems design. Calls to establish means of benchmarking functional models (Summers et al., 2017) and/or introduce a shared functional modelling perspective (Eisenbart et al. 2017) have been made but the research has not yet progressed sufficiently to improve industry practice. Summarising the literature there remain significant challenges to understanding function, resulting in several challenges specifically of concern to the systems engineer, which would not be contested within industry practice:

- The inconsistency within the engineering community to the different meanings of function introduces potential for error in design decision-making if different perspectives working on the same system problem are not viewing the same problem space

- Differing perspectives of function have led to proliferation in tools to produce models of function each of which are valid within their intended domain but are not necessarily used to capture the same view even if from differing viewpoints. Further the tools are often not interoperable i.e., data sharing between the differing tools is not easily accomplished if at all

- Systems engineering is concerned with the whole life cycle not only design concepts. Models used by the systems engineer need to support through life management of the product or system

Acceptance and working with these challenges is essential to the success of any proposed methodology, including MBSE, seeking to exploit function to address the challenges identified here. Traditional functional decomposition, in the form of hierarchical trees only identify some elements of function. Complex designs require a richer understanding, e.g., what functions are dependent upon others? How do functions interact? What conditions are needed to trigger functionality? We need to understand the logical decomposition, and the flow of data between functions to achieve desired product behaviour. 


\subsection{System Architecture}

System architecture concerns the fundamental structure of the product, where functions are mapped to product elements. There are several definitions of system architecture. Ulrich and Eppinger (1995) define the system architecture as "the arrangement of the functional elements into physical blocks." Ulrich (1995) refines this definition further "(1) the arrangement of functional elements; (2) the mapping from functional elements to physical components; (3) the specification of the interfaces among interacting physical components." Crawley (2007) defines the system architecture as "the embodiment of concept and the allocation of physical/informational function to elements of form, and definition of interfaces among elements and with the surrounding context." Sillitto (2014) is more expansive, explaining the purpose of the System Architecture as setting out the "parts of the system, what they do and how they fit and work together". Elaborating the system architecture as describing what is to be designed, the purpose of the architecture he contends is to ensure that all the different elements of the system, once connected and "in their operating environment", fit and work together, achieve the intended purpose of the system, without unacceptable unintended consequence. Whilst Sillitto argues that systems engineering focusses too much on architectural frameworks, he acknowledges that they can provide significant value if they focus on simplicity. He states that a more complex framework should only be developed if it is "absolutely clear that it will add value commensurate with the extra complexity". It is with that in mind that the notion of Reference Architecture is introduced and that a concept of Reference Functional Architecture will be explored in section 3 .

\subsection{Reference Architectures as a means to aid design decision-making in MBSE?}

The concept of Reference Architectures emerged from software and introduced notions of patterns and re-use in architecture. From the systems engineering perspective the software approach seemed to lack a holistic view of the system with no robust definition for the term (Cloutier et al., 2009). The absence of a solid characterization has not prevented industry from adopting the concept, though introduction of the ISO42010 standard for systems and software engineering - architecture description (ISO, 2011) provides standardization for the practise of architecting in systems engineering. Reference architectures are analogous to architecture framework within the ISO42010 standard and are proposed by some MBSE practitioners as a response to complex system design problems. Claims are made (Telleschi, 2018) that these reference architectures improve modularity (i.e., enable packages of 'features' to made available to configure specific solutions) and re-use (mitigate starting from 'scratch' by using existing 'blocks' of architecture) in system design, enabling reduced lead times and improved design decision-making (through earlier detection of defects) and perceived cost avoidance gains in later life cycle stages. The common purpose being to provide guidance for future development (Cloutier, 2009, Behere \& Törngren, 2016).

\section{REFERENCE FUNCTIONAL ARCHITECTURES}

This section discusses the concept of a reference architecture for the functions of a system. Drawing on the descriptions set out in sections 2.4 and 2.5, the following sections explain the concept from a MBSE perspective and introduce an industry example, and how this example was applied to the concept of a hairdryer to analyse the potential wider application of the approach.

\subsection{Functional architecture and related concepts}

Functional architectures are essentially a functional view of the system architecture (Behere \& Törngren, 2016). They are one of several architecture views that comprise the overarching system architecture description in MBSE (Gollaglee, 2020). According to Gollaglee reference architecture is analogous to an architecture framework, i.e., its purpose is to establish common practises by constraining the way in which architecture descriptions are produced (Gollaglee, 2020, ISO 2011). Functional decomposition has long been performed using hierarchical Functional Breakdown Structures these permit only a limited analysis of function. The following sections explore a concept of creating a reference functional architecture using functional chain analysis (Lucero, 2014, Agyemang, 2017, Telleschi, 2018).

\subsubsection{Motivation for developing reference functional architecture}

MBDA Missile Systems, a pan European company that designs and manufactures complex weapons systems, has developed a concept using functional chain analysis to create a reference functional 
architecture to support design re-use (Telleschi, 2018 and, Caroni, 2019). The wider business motivations that led MBDA to develop then adopt this approach were identified as [1] managing increasing complexity in missile design, [2] managing the number of variants in the MBDA product portfolio, [3] better enabling cooperative multinational programs, [4] managing increasing requirements due to increasing demand for missile interoperability, and [5] reduce overall cost through providing modular and interoperable solutions - design re-use (Caroni, 2019). The result was the development by an international team including the first author of what MBDA call the Reference Missile Functional Architecture (RMFA). RMFA was developed from existing missile design concepts with a specific focus on re-use of missile functions across the MBDA missile portfolio. However, a reference functional architecture can be developed from scratch, the motivation being design for future re-use. This approach is being explored by the first author in the development of bio-inspired unmanned air systems.

\subsubsection{The Reference Missile Functional Architecture}

Telleschi (2018) and Caroni (2019) view functional chains as a powerful means to describe functional architecture in complex system and apply functional chains analysis to designing MBDA's complex weapon systems. Caroni (2019) defines a functional chain as a subset of system functions that are isolated so as to be visualised or analysed. Unpacking functions into functional chains enables an analysis of the interaction between different functions through common elements of a functional chain. It is very important to recognise these interactions of functions because they act as change multipliers through which a change to one function and therefore one part of the system potentially affects many others. It is also a means of avoiding a duplication of development effort where the same sub-function is developed multiple times in different ways. Caroni (2019) argues that RMFA is especially useful during complex systems development, enabling understanding of the system and its functionality across multiple specialist teams. The MBSE approach adopted by MBDA for developing RMFA differs from the manner in which functional analysis is typically addressed using hierarchical breakdown structures. RMFA uses the notion of functional chains, which Telleschi (2018) describes as providing "sequenced functions which provide the system with well-defined sub capabilities". Within the RMFA there are 10 functional chains each comprising 10 functions that capture the missile system functional behaviour. The activation of a functional chain is dependent on time (e.g., evolution of the missile mission) and mapped by allocating each function to a missile state for each missile use case (Caroni, 2019). The RMFA allows functionality to be reused directly though it also permits functionality to be tailored through the tuning of the function parameters. The following sections summarise the application of the RMFA in the conceptual design of a simple product, a hairdryer. The aim is to use MBSE concepts, and the Systems Modelling Language (SysML) notation, and the RMFA functional chain approach to conceptually explore the utility of the approach in aiding design decision-making and identify opportunities for further research.

\subsection{Applying the RMFA approach through a worked example using a hairdryer}

MBDA's Reference Missile Functional Architecture (RMFA) uses use case diagrams to identify missile needs. These use cases identify the high-level function for any missile product, for example, Perform Navigation (Caroni, 2019). The use cases are tailored to meet specific solution needs (e.g., air defence, ground attack) and describe [1] the use case goal, [2] a primary actor, i.e., the actor that initiates the interaction with the missile, [3] pre-conditions that should be met before the use case can commence, [4] the initiator, [5] the perceived functionality, and [6] the condition the missile is in after use case success . For the hairdryer concept we have taken the actor to be a person who wishes to use the hair dryer to achieve the goal: dry hair. The pre-condition is taken to be the hairdryer is connected to a suitable power source (e.g., it is plugged in) and the trigger is a user command by means of a control switch to turn the hairdryer on. Therefore, the high-level function for the hairdryer to perform is simply: Dry Hair. The successful post-condition is the user has dry hair. Thus far the RMFA provides no more than a well defined MBSE approach to their product design, albeit using a language specific to the MBDA product domain, complex missile systems.

Using a traditional MBSE approach both black box and white box levels RMFA identifies components of functionality to be re-used in both missile and missile sub-systems. For our hairdryer concept this situates the functional elements and information/dataflows between functional elements, identifying 3 system functions, Figure 1. An interesting feature of the RMFA approach is the ability to tailor, or tune, RMFA function parameters for a specific missile system need without modifying the RMFA. 
This approach takes the RMFA as a reference and using a standard SysML generalisation relationship and populating that with the detail of the specialized requirement. This ability is explained in Telleschi (2018) and an illustrated example is provided in Caroni (2109) but was not explored using the hairdryer concept.

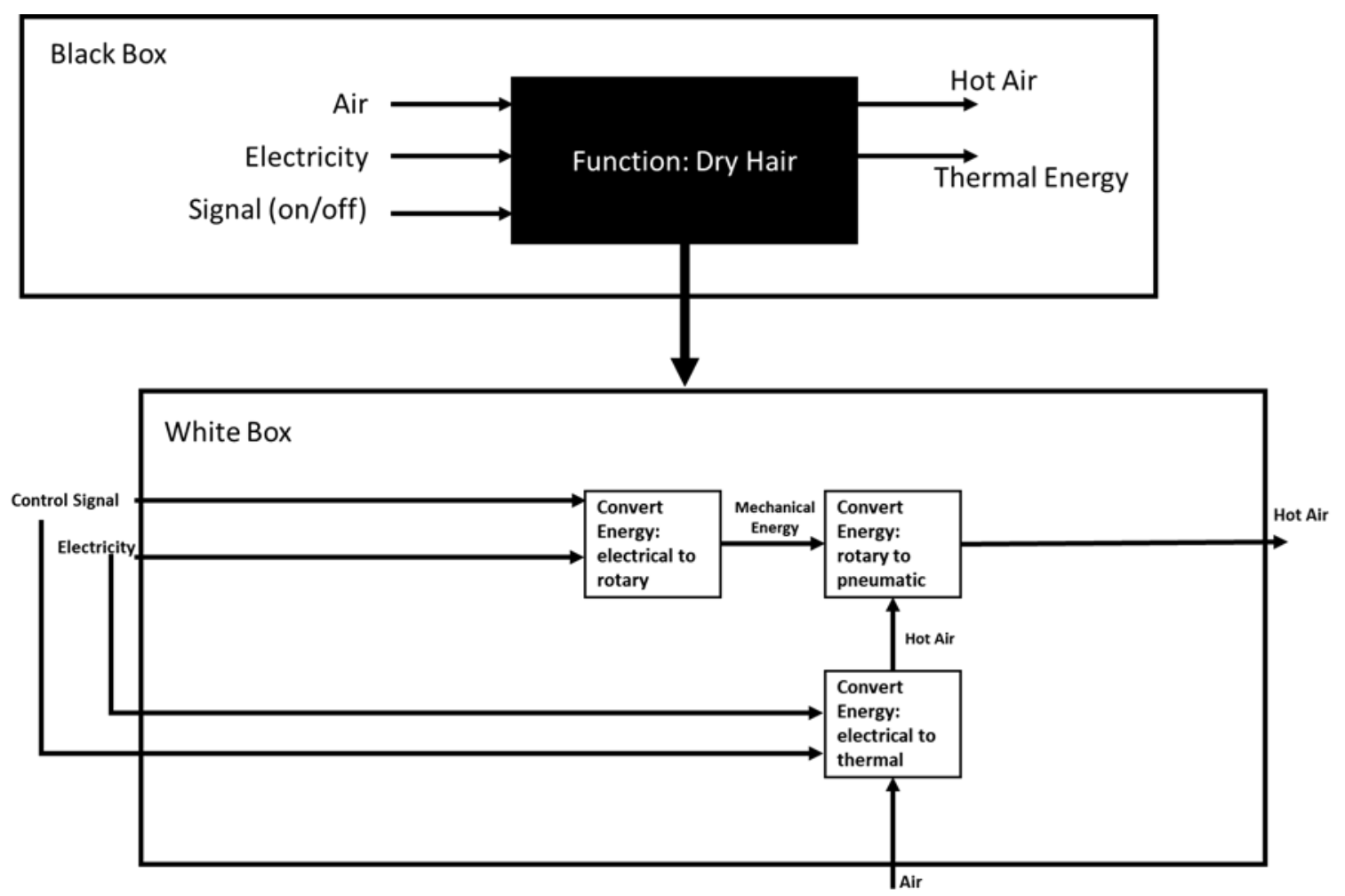

Figure 1. Traditional black box \& white box analysis for the hairdryer concept

\subsubsection{Application of RMFA Functional Chain Analysis to the hairdryer concept}

The functional chain analysis process used in developing, and utilised when using, the RMFA is not part of a typical MBSE approach, as the tools currently in use in MBDA, as with many other organisations, do not currently support modelling functions in this way. Functional chain analysis advocated by Telleschi and Caroni is undertaken by constructing a matrix for each use case to describe the relationship between function and state. Applied to the hairdryer concept this process was undertaken by constructing a lattice like diagram illustrated in both Telleschi (2018) and Caroni (2019) to allocate a set of possible hairdryer functions to a set of nominal hairdryer states, Figure 2.

The aim in conducting functional analysis in this manner is to better identify functional and nonfunctional requirements attached to the function which will in turn, better support elicitation of subsystem requirements once the functions are allocated to a specific sub-system. Telleschi claims the lattice like structure produced by modelling function in this way has been successfully utilized within MBDA's complex system development projects to facilitate better understanding of the system under development and its functionality across multiple specialist teams. A further advantage of this method is the facilitation of separating the mission life cycle of a complex system into time related phases, for MBDA this resulted in development of a taxonomy for missile phases as a result of applying this phase related functional chain approach. Dependency between functions and elements of function are easier to identify. For example, it can clearly be seen in Figure 2 that [1] Provide air, and [2] Provide heat are mutually dependent on [3] Provide power. This may seem obvious in the case of our simple hairdryer however, for a complex system such as a missile with more than 10 functional chains each having at least 10 functions in each chain with a mission life cycle consisting of multiple states and phases, the value of the approach starts to become interesting. 


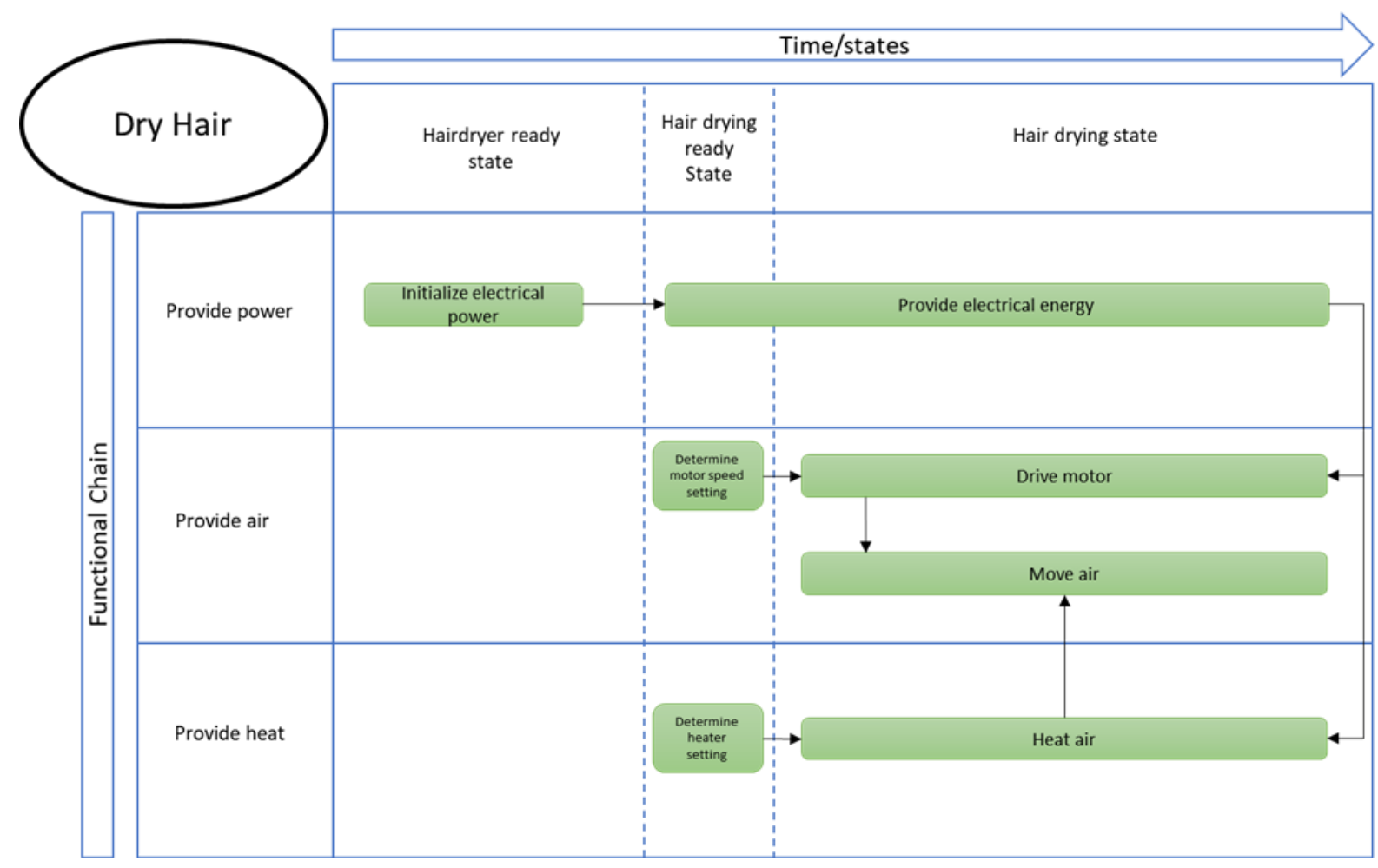

Figure 2. Example association between functional chains for the use case: Dry hair

Once the functional chain analysis is complete, we now consider the composition of the embodiment, the physical architecture of the hairdryer and how the functions might be allocated to a physical architecture. Neither Telleschi (2018), nor Caroni (2019) discusses specifically the functional to physical allocation, however, it is noted that using the functional chain analysis approach enables deliberation without constraining the design to a specific embodiment, Figure 3. For our hairdryer this facilitates consideration of several potential embodiments, Figure 4.

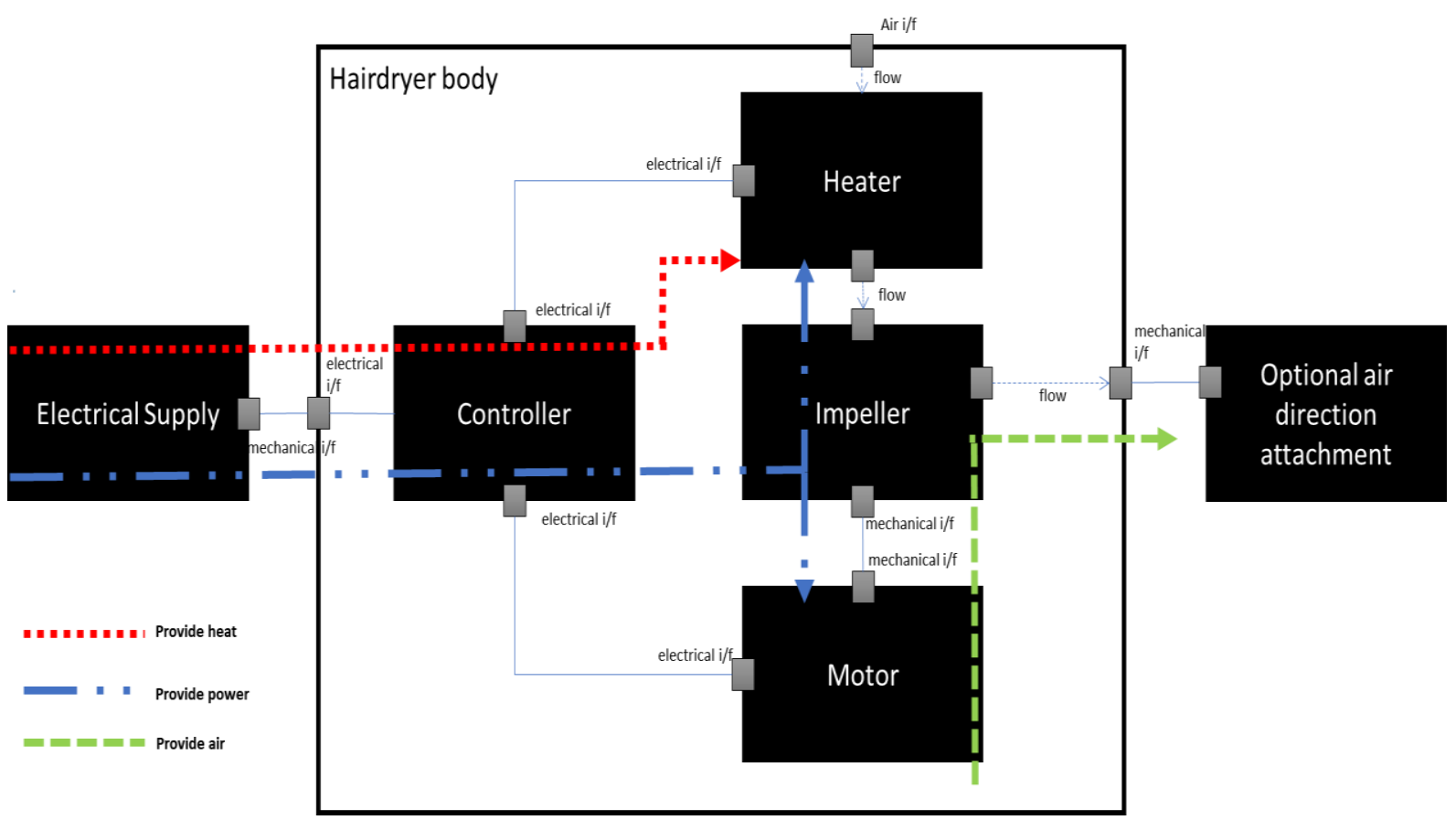

Figure 3. Functional chain allocation to physical architecture 

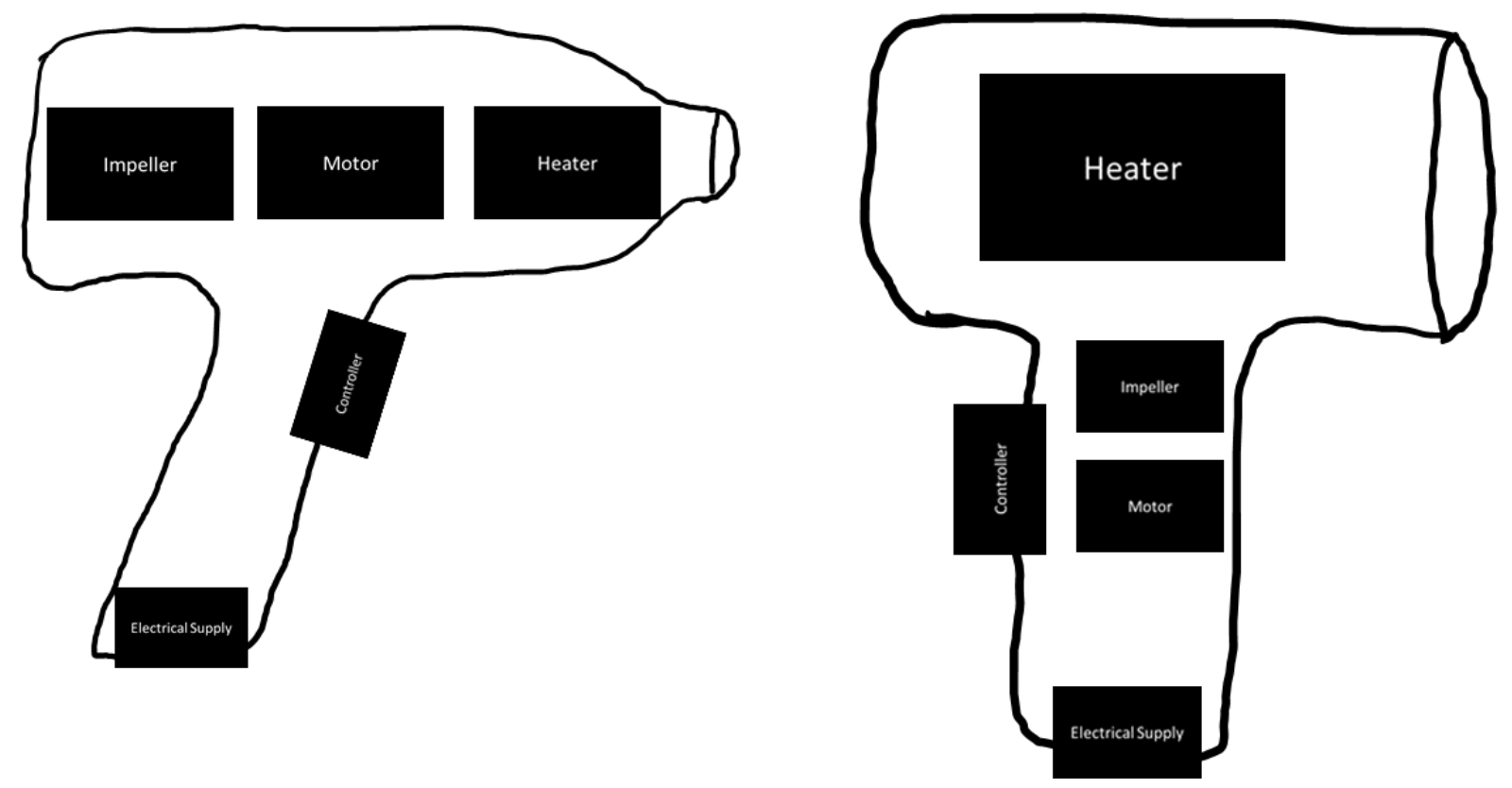

Figure 4. Potential embodiments

\section{DISCUSSION \& RESEARCH RECOMMENDATIONS}

Section 2.4 considered system architecture noting for the systems engineer the system architecture is more than a functional to physical allocation (Sillitto, 2014, Gollaglee, 2020). Motivations for adopting the reference functional architecture approach were given in 3.1.1, whilst illustrating MBDA's specific motivations these appear similar to many in industry, not only defence. Although the MBDA terminology can be difficult to understand, RMFA is a good industry example of the application of MBSE to creating a reference architecture for the development of a complex product. In this context it appears to fall foul of Sillitto's requirement for simplicity however, it does appear that to MBDA the value is commensurate with the added complexity. As used in the hairdryer concept, and for any simple system, there would be little value in developing a reference architecture and none at all in developing something as complex as an RMFA type architecture. As mentioned, the MBDA terminology is not easy to grasp, and significant explanation was needed in applying to the hairdryer concept. In respect of MBSE applied to the hairdryer concept there is of course no significant challenge other than to perhaps question the value of applying MBSE to such a simple product. The major limitations identified through the application of the approach to a case study from a different domain are; [1] beyond the scope of this study, it is important to understand the merits of applying MBSE itself in the context of the complexity of the product to be designed (e.g., cost of education and training people, tool acquisition, development of the framework for reference, implementation, ongoing support, change control), [2] to translate the terminology used by MBDA in the RMFA into terminology understood in the domain to which it would be applied, and [3] understanding how to fuse the understanding and insights gained from the functional chain analysis and ensuring these are actually captured in the model of the specific product. The functional chain approach appears relatively simple when applied to the hairdryer example however, functional chain analysis is not unique to RMFA, this requires further research and understanding to explore whether the RMFA approach is more effective and cross-domain applicable than alternatives. Functional chain analysis as applied here, results in a lattice like structure that does appear to afford multiple system architectures which may offer significant advantages over other more traditional approaches. For example, we could more clearly identify the dependencies between functions and elements of function in the lattice than we might have been able using a traditional hierarchical breakdown structure method. Further research using more complex examples will be required to underpin this with a good degree of confidence. Though not applied in the hairdryer example, the RMFA authors claim enablement of a common taxonomy (in the case of RMFA a taxonomy for missile systems) that can be shared across multiple project teams. The potential for a generic taxonomy for reference functional architectures will require further research if the concept is to be more widely utilised or they may remain specific to industry 
domains. Analysis of this brief exercise exposes limitations with current functional decomposition approaches such as functional breakdown structures. Most significantly, their hierarchical nature does not facilitate exposure of interdependency between functions. The lattice like structure of functional chain analysis does appear to better facilitate further and deeper understanding, e.g., functional chains analysis can aid identification of potential failure points thorough consideration of the relationships between functions, functional chains, and the phases of operation of the system. E.g., if the functional chain; Provide Heat, were activated prior to the functional chain; Provide Power there is potential for overheating of the heater and failure. Functionality modelled using this approach is not constrained to any specific physical embodiment, therefore it avoids premature commitment to physical design constraints thus affording better design decision-making. Potentially, reference functional architectures developed using this approach can be re-used many times over if the required functionality of the desired system/product is broadly similar. It is recommended that further research is conducted to; [1] explore the application and utility of the RMFA approach when applied to a complex product from a non-defence domain, [2] understand the rigour of the functional chain analysis approach described here and the alternatives, and [3] consider the possibility of a generic taxonomy for reference functional architectures that can apply across multiple domains.

\section{CONCLUSIONS}

Reference functional architectures have been proposed to support design re-use by describing product functions using functional chains that describe functional architecture in complex system . RMFA at one level is simply an architectural framework used as a reference for the development of a complex product. As shown in the example presented in this paper, the concept could be applied to any product or system, though it is recommended that Sillitto's simplicity concept is taken on board before doing so. The functional chain concept appears to have wider utility than traditional functional decomposition approaches in considering the relationship between functions and embodiment. However, wider adoption requires development of a common taxonomy across multiple disciplines, even if practising in the same domain. Further research is required to consider potentially related approaches to identify commonality, or we may lose the opportunity to address some of the challenges associated with function, modelling and MBSE.

\section{REFERENCES}

Agyemang, M., Linsey, J., and Turner, C. (2017) "Transforming functional models to critical chain models via expert knowledge and automatic parsing rules for design analogy identification" AI EDAM, Vol 31 No.4, pp. 501-511, https://dx.doi.org/10.1017/S0890060417000488

Behere, S., and Törngren, M. (2016). "A functional reference architecture for autonomous driving”, Information and Software Technology, Vol 73, pp. 136-150, https://doi.org/10.1016/j.infsof.2015. 12.008

Box, G. E. P. (1976), "Science and statistics”, Journal of the American Statistical Association, Vol 71 No.356, pp. 791-799, https://dx.doi.org/10.1080/01621459.1976.10480949

Box, G. E. P. (1979), "Robustness in the strategy of scientific model building", Robustness in Statistics, pp. 201236, https://dx.doi.org/10.1016/B978-0-12-438150-6.50018-2,

Caroni, A., Telleschi, G., Mactaggart, I., Willingham, E. and Pradel, P-H. (2019), "Reference Functional Missile Architecture: An Architecting Toolset to Drive Design and Modelling", REConf, Munich, 11-15 March 2019, Hood Group, Munich.

Cloutier, R., Muller, G., Verma, D., Nilchani, R., Hole, E. and Bone, M. (2009), "The Concept of Reference Architecture", Systems Engineering, Vol. 13 No. 1, pp. 14-27. https://dx.doi.org/10.1002/sys.20129

Crawley, E.F. (2007). ESD.34 Systems Architecting_Lecture Notes. Cambridge, MA: MIT Engineering Systems Division.

Crilly, N. (2013). "Function propagation through nested systems". Design Studies, Vol. 34 No.2, pp. $216-242$.

Eckert, C. (2013). "That which is not form: the practical challenges in using functional concepts in design". AI EDAM, Vol. 27 No.3, pp. 217-231

Eisenbart, B., Mandel, C., Gericke, K. \& Blessing, L. (2013) “An Analysis of Functional Modelling Approaches Across Disciplines”. AI EDAM, Vol. 27 No.3, pp. 281-289.

Eisenbart, B., Gericke, K., \& Blessing, L. (2017) "Taking a look at the utilisation of function models in interdisciplinary design: insights from ten engineering companies", Research in Engineering Design, Vol 28, pp.299-331. https://dx.doi.org/10.1007/s00163-016-0242-3 
Friedenthal, S., Moore, A., Steiner, R. (2012) A Practical Guide to SysML: The Systems Modeling Language. MK/OMG Press. Waltham, USA.

Gero, J. and Kannengieser, U. (2004). “The situated Function-Structure-Behaviour Framework”. Design Studies, Vol 25, No. 4 pp.373-391.

Gollaglee, J., McNaughton, B., Plum, N., Powley, S. and Steponkute, U. (2020), “The Challenging Landscape of Model-based Initiatives", ASEC20, 17-18 November 2020, INCOSE UK Ltd., London, pp. 53-61.

Hand, D. J. (2014), “Wonderful examples, but let's not close our eyes”, Statistical Science, Vol. 29, pp. 98-100, https://dx.doi.org/10.1214/13-STS446

Holt, J. \& Perry, S. (2013). SysML for Systems Engineers: A Model Based Approach. 2nd Edition. Institution of Engineering and Technology. Stevenage, UK.

INCOSE (2014). A World in Motion - The INCOSE 2025 vision. INCOSE. San Diego, USA.

INCOSE (2015). Systems Engineering Handbook: A Guide for System Life Cycle Processes and Activities (4th ed.). John Wiley \& Sons, Inc. Hoboken, USA.

ISO (2011). ISO/IEC/IEEE 42010:2011 Systems and software engineering - Architecture description. Available on-line at: (ISO-ISO/IEC/IEEE 42010:2011-Systems and software engineering - Architecture description), Accessed: (18 October 2020).

Krob, D. (2017), CESAM: CESAMES System Architecting Method: A Pocket Guide [online] CES AMES. Available at: (http://cesam.community/en/cesam/), Accessed: (18 May 2020).

Lucero, B., Viswanathan, V.K., Linsey, J.S., and Turner, C.J. (2014). "Identifying Critical Functions for Use Across Engineering Design Domains” Journal of Mechanical Design, Vol. 136 Issue 12. https://dx.doi.org/10.1115/1.4028280

Madni, A.M. and Purohit, S. (2019) "Economic Analysis of Model-based Systems Engineering”. Systems, Vol. 7 No.12. https://dx.doi.org/10.3390/systems7010012

Maier, J.F., Eckert, C.M., and Clarkson, J. (2016). Model granularity in engineering design - concepts and framework. Des. Sci., Vol 3, e1. Cambridge University Press. Available at: https://www.cambridge. org/core/journals/design-science/article/model-granularity-in-engineering-design-concepts-andframework/8F282B38679B56287E4B4DF861EF33F0 (Accessed 16 November 2019).

Pahl, G. and Beitz, W. (2007). Engineering Design: A Systematic Approach - 3rd Edition. Springer-Verlag. London.

Sillitto, H. (2014). Architecting Systems: Concepts, Principles and Practice. College Publications. United Kingdom.

Suh, N.P. (2001). Axiomatic Design: Advances and Applications. Oxford University Press. United Kingdom.

Summers, J. D., Eckert, C., \& Goel, A. K. (2017). "Function in engineering: benchmarking representations and models". AI EDAM, Vol.31 No.4, pp.401-412. https://dx.doi.org/10.1017/S0890060417000476

Telleschi, G., Caroni, A., Willingham, E. and Pradel, P-H. (2018), "Reference Functional Missile Architecture: addressing design in a multi-national defence company". CIISE 2018, pp. 31-37

Ulrich, K. (1995). The role of product architecture in the manufacturing firm. Research Policy 24(3), 19-440.

Ulrich, K., \& Eppinger, S.D. (1995). Product Design and Development. New York: Irwin McGraw-Hill.

Vermaas, P. E. (2009). “The Flexible meaning of Function in Engineering”. ICED09. Stanford, USA. 24-27 August 2009, Vol. 2, pp. 113-124.

Vermaas, P.E. (2010). "Technical functions: towards accepting different engineering meanings with one overall account”. TMCE Symp 2010. Ancona Italy. 12-16 April 2010, pp. 183-194.

Vermaas, P. E. (2013). "The coexistence of engineering meanings of function: four responses and their methodological implications". AI EDAM, Vol. 27 No. 3, pp. 191-202.

Weilkiens, T. (2006). Systems Engineering with SysML, MK/OMG, MA USA. 